Tho variation of $\alpha$ with $E$ and $P$ in the alkyl chlorides is expressed accurately by the functional form $\alpha / P=A \exp (-B P / E)$, as is illustrated in Fig. 1. It is especially to be noted that the functional form $\alpha / E=A \exp (-B P \mid E)$ will not fit the data. Values of the constants $A$ and $B$, as derived from the curves in Fig. 1, are listod in Table 1.

\begin{tabular}{|c|c|c|c|c|}
\hline Gas & $\stackrel{A}{A} .^{-1} \mathrm{~mm}^{-1}$ & $\begin{array}{c}\text { Table 1 } \\
\stackrel{B}{v} \mathrm{~cm} \cdot \cdot^{-1} \mathrm{~mm} .^{-1}\end{array}$ & $\begin{array}{l}\varepsilon i \\
\mathrm{eV}\end{array}$ & $\begin{array}{c}B / \varepsilon i^{3 / 2} \\
\mathrm{~cm} \cdot{ }^{-1} \mathrm{~mm} .^{-1} v^{-1 / 2}\end{array}$ \\
\hline $\begin{array}{l}\mathrm{CH}_{3} \mathrm{Cl} \\
\mathrm{C}_{2} \mathrm{H}_{8} \mathrm{Cl} \\
n-\mathrm{C}_{3} \mathrm{H}_{7} \mathrm{Cl} \\
n-\mathrm{Cl}_{3} \mathrm{H}_{9} \mathrm{Cl} \\
n-\mathrm{C}_{5} \mathrm{H}_{11} \mathrm{Cl}\end{array}$ & $\begin{array}{l}13 \cdot 4 \\
16 \cdot 1 \\
20 \cdot 7 \\
28 \cdot 4 \\
40 \cdot 4\end{array}$ & $\begin{array}{l}244 \\
325 \\
388 \\
471 \\
\mathbf{5 4 9}\end{array}$ & $\begin{array}{l}11 \cdot 28^{*} \\
10.97^{*} \\
10 \cdot 8 \\
10 \cdot 7 \\
10 \cdot 6 \neq\end{array}$ & $\begin{array}{r}6 \cdot 4 \\
8 \cdot 9 \\
10 \cdot 9 \\
13 \cdot 4 \\
15 \cdot 8\end{array}$ \\
\hline
\end{tabular}

At present there exists no exact theory for the correlation of these quantities with molecular structure. The approximate theory of Crowe, Bragg and Devins ${ }^{3}$ predicts that the constant $B$ will vary with molecular parameters in a homologous series as $B=C \varepsilon_{i}{ }^{3 / 2} \theta$, where $\varepsilon_{i}$ is the molecular ionization energy and $\theta$ the total cross-section for olectron scattering. Devins and Crowe ${ }^{4}$ and Heylen and Lewis ${ }^{5}$ dorived values of $B$ for a number of saturated hydrocarbon gases from sparking potential measurements. They found that $B / \varepsilon_{i}^{3 / 2}$ is directly proportional to the number of carbon-hydrogen bonds in the $n$-alkane gases, indicating that electrons in the carbon-hydrogen bonding orbitals (that is, the eloctrons in the outermost molecular orbitals) are most effective in electron scattering.

The values of $B / \varepsilon_{i}^{3 / 2}$ of Devins and Crowe for the alkanes are plotted in Fig. 2 along with our values for the alkyl chlorides. From the theory, the slope of the alkyl chloride curve is proportional to the $\mathrm{C}-\mathrm{H}$ bond cross-section, while the intercept is proportional to the cross-section of chlorine bonded to a carbon atom. Analysis of the data yields a loast-squaros line of slope $1.16 \pm 0.03$ and intercept $2.97 \pm 0.07$. The slope for the alkyl chloride data is approximately twice that for the alkanes. The largest part of this difference is possibly due to an incorrect choice for the functional form for $\alpha$ by Devins and Crowe. The sparking potential data cover a rather limited range of $E / P$, so that their data can be fitted equally well using eithor $\alpha / P=$ $A \exp (-B P / E)$ or $\alpha / E=A \exp (-B P / E)$. The $B$ values they quote were obtained using the latter form. If the former expression were used instead, all of the $B$ values for the alkanes would be larger by a multi-

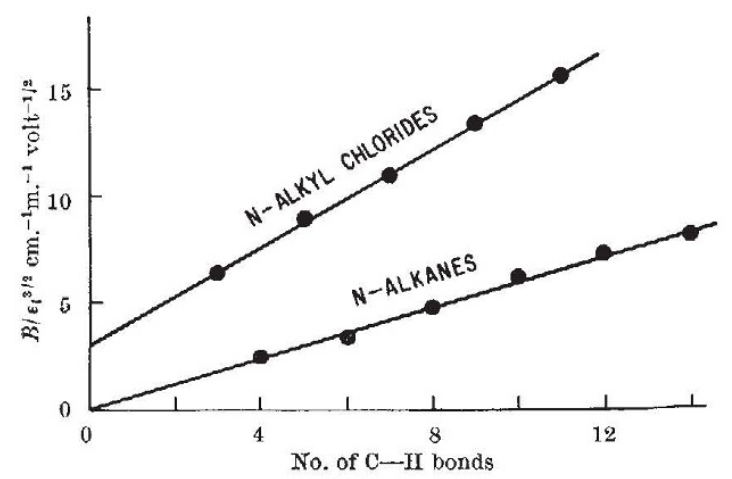
Fig. 2. Dependence of $B / \varepsilon_{i}^{3 / 2}$ on the number of carbon-hydrogen
bonds per molecule. Upper curve, this work; lower curve, ref. 4 plicative constant of approximately 1.5. Even so, the two slopes in Fig. 2 would still be different by 25 per cent, indicating either a larger $\mathrm{C}-\mathrm{H}$ bond cross-section in the alkyl chlorides or different proportionality constants for the two homologous serios in the equation $B=C \varepsilon_{i}^{3 / 2} \theta$.

The constant $A$ in the alkyl chlorides appears to vary as $a+b\left(n_{\mathrm{C}-\mathrm{H}}\right)^{3}$. The significance of this observation is not clear.

We are indebted to E. M. Hadsell for performing analyses and purifications by vapour phase chromatography.

$$
\begin{aligned}
& \text { J. C. Devins } \\
& \text { O. H. LeBland, JUN. }
\end{aligned}
$$

General Electric Research Laboratory, Schonectady, New York.

${ }^{1}$ Geballe, R., and Harrison, II. A., Phys. Rev., 91, 1 (1953).

2 Dibeler, V. H., and Reese, R. M., J. Res. Nat. Bur. Standards, 54, 127 (1955).

${ }^{3}$ Crowe, R. W., Bragg, J. K., and Devins, J. C,, J. App. Phys., 26, 1121 (1955).

${ }^{4}$ Devins, J. C., and Crowe, R. W., J. Chem. Phys., 25, 1053 (1950)

'Heylen, A. E. D., and Lewis, T. J., Brit. I. App. Phys., 1, 411 $(1956)$.

\section{Homogeneous Synthesis of Isotactic Polystyrene using $n$-Butyllithium Initiator}

ORGANOLITHIUM compounds are known to cause the stereospecific polymerization of dienes ${ }^{1}$ and methacrylates ${ }^{2}$. Via $n$-butyllithium, styrene polymorizes easily to atactic polymer at ordinary temperatures ${ }^{3,4}$. We have now found that styrone may be polymerized at low temperatures, employing butyllithium as a soluble initiator, to crystallizable isotactic polystyrene.

Polystyrene prepared in a hydrocarbon medium at temperatures in the range of $-30^{\circ} \mathrm{C}$. and below oxhibits infra-red, X-ray diffraction and nuclear spin resonance spectra identical to those shown by isotactic crystalline polystyrene prepared via triethyl-aluminium-titanium trichloride catalysis. In the presence of ethers, however, only atactic polymer forms, even at $-78^{\circ} \mathrm{C}$. Isotactic polymerization is much slower at $-40^{\circ}(\sim 5$ per cent conversion in $48 \mathrm{hr}$.) in a $7: 1$ volume ratio styrene-toluene mixture than at $-78^{\circ}$ in tetrahydrofuran (>90 per cent conv. in $1 \mathrm{hr}$.$) . Butyllithium concentrations of 5-10 m.mol./1.$ were used.

A block copolymer of atactic and isotactic types of polystyrene may be prepared by adding an other to a styrene polymerization proceeding at $-40^{\circ}$ in toluene. The same result may be achieved by raising the temperature to $0^{\circ}$ after polymerizing for a period of time at about $-40^{\circ}$. Blocks of a second monomer species (such as isoprene, alkyl mothacrylatos and acrylonitrile) on isotactic polystyrene can be prepared even though styrene conversion is far from complet $\theta^{5}$.

Monsanto Chomical Co.,

\section{R. J. KERN}

\section{Dayton 7, Ohio. June 20.}

${ }^{1}$ Stearns, R. S., and Forman, L. E., J. Polymer Sci., 41, 381 (1959). ${ }^{2}$ Fox, T. G., Garrett, B. S., Goode, W. E., Gratch, S., Kincaid, J. F', ${ }^{3}$ Welch, F. J., J. Amer. Chem. Soc., 81, 1345 (1959).

${ }^{4}$ O'Driscoll, K. F., and Tobolsky, A. V., J. Polymer Sci., 35, 259 (1959).

s Graham, R. K., Dunkelberger, D. L., and Goode, W. E., J. Amer. Chem. Soc., 82, $400(1980)$. 